\title{
The effect of temperature on different Salmonella serotypes during warm seasons in a Mediterranean climate city, Adelaide, Australia
}

\author{
A. MILAZZO ${ }^{1 *}$, L. C. GILES ${ }^{1}$, Y. ZHANG ${ }^{1,2}$, A. P. KOEHLER ${ }^{3}$, J. E. HILLER ${ }^{1,4}$ \\ AND P. BI ${ }^{1 *}$ \\ ${ }^{1}$ School of Public Health, The University of Adelaide, Adelaide, SA, Australia \\ ${ }^{2}$ School of Public Health, The University of Sydney, Sydney, NSW, Australia \\ ${ }^{3}$ Communicable Disease Control Branch, Department for Health and Ageing, Adelaide, SA, Australia \\ ${ }^{4}$ School of Health Sciences, Swinburne University of Technology, Melbourne, VIC, Australia
}

Received 18 April 2015; Final revision 18 August 2015; Accepted 1 October 2015; first published online 2 November 2015

\section{SUMMARY}

Changing trends in foodborne disease are influenced by many factors, including temperature. Globally and in Australia, warmer ambient temperatures are projected to rise if climate change continues. Salmonella spp. are a temperature-sensitive pathogen and rising temperature can have a substantial effect on disease burden affecting human health. We examined the relationship between temperature and Salmonella spp. and serotype notifications in Adelaide, Australia. Timeseries Poisson regression models were fit to estimate the effect of temperature during warmer months on Salmonella spp. and serotype cases notified from 1990 to 2012. Long-term trends, seasonality, autocorrelation and lagged effects were included in the statistical models. Daily Salmonella spp. counts increased by $1 \cdot 3 \%$ [incidence rate ratio (IRR) $1 \cdot 013,95 \%$ confidence interval (CI) $1.008-1 \cdot 019]$ per $1{ }^{\circ} \mathrm{C}$ rise in temperature in the warm season with greater increases observed in specific serotype and phage-type cases ranging from $3 \cdot 4 \%$ (IRR $1 \cdot 034,95 \%$ CI $1 \cdot 008-1 \cdot 061$ ) to $4 \cdot 4 \%$ (IRR $1 \cdot 044,95 \%$ CI 1.024-1.064). We observed increased cases of $S$. Typhimurium PT9 and $S$. Typhimurium PT108 notifications above a threshold of $39^{\circ} \mathrm{C}$. This study has identified the impact of warm season temperature on different Salmonella spp. strains and confirms higher temperature has a greater effect on phage-type notifications. The findings will contribute targeted information for public health policy interventions, including food safety programmes during warmer weather.

Key words: Climate - impact of, foodborne infections, infectious disease epidemiology, Salmonella, Salmonella typing.

\section{INTRODUCTION}

Increases in ambient temperature can have a substantial effect on the burden of infectious diseases with

\footnotetext{
* Author for correspondence: Ms. A. Milazzo or Professor P. Bi, School of Public Health, Level 7, 178 North Terrace, Adelaide, SA 5000, Australia.

(Email: adriana.milazzo@adelaide.edu.au)

(Email: peng.bi@adelaide.edu.au)
}

some, such as salmonellosis, following a seasonal pattern suggestive of an association with higher temperature [1]. Human Salmonella spp. infection is a significant global public health problem with an estimated 93.8 million cases per year, of which 80.3 million are foodborne [2]. The incidence of infectious gastroenteritis attributed to foodborne disease in Australia, with a population of about 22 million, is estimated to be $4 \cdot 1$ million cases per year [3]. From 
2007 to 2011 the mean rate of salmonellosis per 100000 population in South Australia (SA) was 48.4 compared to $46 \cdot 6$ for Australia [4]. In 2009, 34\% of the foodborne outbreaks reported in Australia was caused by Salmonella spp. infection [5], which is substantial given that it is a commonly notified cause of infectious gastroenteritis [6]. If the effects of extreme weather events, including higher temperature in the warm season continue to rise there could be an upsurge in incidence of salmonellosis adding to the global burden currently associated with Salmonella spp. infection [7].

The average temperature in Australia has been rising over the last 50 years [7]. In Adelaide, the capital city of SA and location for the present study, the average yearly temperature has risen by $1.1{ }^{\circ} \mathrm{C}$ over the same time period [8]. Adelaide experiences a Mediterranean climate with cool wet winters and hot dry summers with higher temperatures in the warm season becoming more frequent, intense and of longer duration [9].

It is well established that survival and multiplication of salmonellosis in the environment and in food is influenced by high temperatures [10]. Previous studies have found an association between warmer ambient temperature and Salmonella spp. infection [11-19]. In spite of this, there is limited information on whether there is greater sensitivity to increased temperature and likelihood of causing foodborne disease among different serotypes of Salmonella spp. Although studies have reported an association with temperature $[20,21]$ and with risk factors and temperature [22] at the serotype level, none have assessed if there is an association with different Salmonella spp. phage types. Considering that there are over 2500 Salmonella spp. serotypes, and over 300 phage types [23], knowing which strains may be linked to warmer temperature will provide a better understanding of the route of transmission of infection.

Not all serotypes cause human disease as some are host adapted to other animal species [24]. Furthermore, the distribution of serotypes and phage types varies both within and between countries [25]. Some may be endemic in the environment and might be associated with environmental routes of transmission such as contact with native animals and drinking untreated water [26] that could become more problematic with increasing warmer temperature [1]. Establishing if there is a relationship with warmer temperature and certain serotypes and phage types will provide valuable information for future public health interventions on foodborne disease prevention during hot weather with specific strategies focused on adaptation.
The aim of this study was to examine if there is a relationship between temperature during the warm season and number of Salmonella spp. notifications, and to examine if there is serotype- and phage-type-specific variation with Salmonella spp. infection in Adelaide. This study has increased relevance with episodes of extreme heat becoming more frequent adding to the increased risk of foodborne disease. Findings from this study will contribute to an understanding of the association of temperature and health outcomes which may be useful when modelling complex climate-health patterns.

\section{METHODS}

\section{Data collection}

\section{Notifiable disease data}

Daily laboratory-confirmed salmonellosis cases resident in the Adelaide metropolitan area and notified to the Communicable Disease Control Branch (CDCB), SA Department for Health and Ageing between 1 January 1990 and 31 December 2012 were obtained from the CDCB notifiable disease surveillance system. Information on date of onset of illness and demographic characteristics was extracted along with the Salmonella spp. serotype and phage type identified for each case.

\section{Climate data}

Climate data from 1990 to 2012 were obtained from an Australian Bureau of Meteorology (BOM) weather monitoring station close to the Adelaide city centre. Daily records were obtained for maximum temperature $\left(T_{\max }\right)$ and mean minimum temperature in degrees Centigrade $\left({ }^{\circ} \mathrm{C}\right)$, rainfall in millimetres $(\mathrm{mm})$, and relative humidity at 09:00 and 15:00 hours in percentage. $T_{\max }$ was used as it is considered to be a better index of exposure than average and mean minimum temperature, and allowed for comparison of our findings with the results from other studies in Adelaide that also used $T_{\max }$ as a predictor of heat-related health outcomes [27]. BOM records from this city monitoring station have been used in previous research conducted in Adelaide and provide an accurate representation of weather conditions across the metropolitan region $[18,28]$.

\section{Statistical analysis}

As the focus of the investigation was on the effects of warmer temperature on the number of Salmonella spp. 
notifications, only data for the warm seasons (1 October-31 March) were included in the analyses. To estimate the effect of $T_{\max }$ in the warm season on daily salmonellosis and specific serotypes we applied a time-series Poisson regression model. The exposure variable was daily $T_{\max }$ with the outcome variable the number of Salmonella spp. notifications reported on each corresponding day during the warm seasons. Daily counts of salmonellosis and specific serotypes recorded during the study period were merged with daily weather records. Salmonella spp. serotypes were selected for analysis based on the five ranked with the highest frequency over the study period. Salmonella spp. cases linked to outbreaks were identified from the CDCB notifiable disease surveillance system and were excluded from the analysis as the relationship of temperature may be different for those with a common exposure compared to sporadic cases with no identified source of infection $[12,18,20]$.

Spearman's correlation was used to examine the relationship between $T_{\max }$ and the daily number of Salmonella spp. notifications, including serotypes over the study period warm seasons. To identify any lagged effects of $T_{\max }$ on daily Salmonella spp. notifications, cross-correlation analyses were performed. Sensitivity analyses of different lag times up to 28 days were conducted based on the cross-correlation results and from existing knowledge on transmission of Salmonella spp. infection. We controlled for autocorrelation (AC) of the outcome variable of daily Salmonella spp. and specific serotype notifications based on the autocorrelation function (ACF), and partial autocorrelation function (PACF) to identify the autoregressive (AR) order and to account for the serial relationship between past and current cases.

Long-term trends in disease incidence and seasonal patterns are potential confounders in examining short-term effects of temperature on notifications of Salmonella spp. infection. To account for these potential confounders in the time-series Poisson regression model we included: day of the week using a categorical variable with Sunday as the reference day, an indicator variable for public holidays, and a year variable (to include all the months in a full warm season within each financial year, i.e. July to June) adjusting for long-term trends. We examined the effect of year in our model to not only account for long-term trends but also changes to the disease notification system. A quadratic term for temperature was included in the statistical models to allow for a nonlinear relationship between daily notifications and $T_{\max }$. Seasonal fluctuations were also controlled for through the restriction of analyses to the warm season each year across the study period.

Lag values were included in the regression models to estimate the delayed effects of temperature on daily Salmonella spp. notifications. Relative humidity and rainfall were excluded because Adelaide experiences dry, hot weather during the warmer months with little rainfall and humidity [18]. Goodness-of-fit tests were used to assess model fit. In the case of overdispersion a negative binomial model was fitted. Incidence rate ratios (IRRs) with 95\% confidence intervals (CIs) are reported with results interpreted as percent (\%) change in the number of daily Salmonella spp. counts per $1{ }^{\circ} \mathrm{C}$ increase in $T_{\max }$ [27].

To examine if any relationship differed above a certain temperature for Salmonella spp. counts and for the serotypes ranked with the highest frequency notified in the warm season, we also investigated the effect of temperature thresholds. We assessed the exposure-response relationship between counts of salmonellosis (or serotypes) and $T_{\max }$ by use of a non-parametric regression lowess (locally weighted scatter plot smooth) smoother at a bandwidth of 0.8 (using $80 \%$ of the data). The plots were visually inspected for temperature thresholds. Piecewise linear regression models were fitted with a single breakpoint at the identified temperature thresholds using the 'hockey-stick' nl command in Stata. Separate piecewise linear regression models (if appropriate) were fitted to examine the effects of temperature in the warm season on daily Salmonella spp. counts and on each of the five serotype counts.

A significance level of 0.05 was accepted for all statistical tests. Analyses were conducted using StataSE v. 13 (StataCorp LP, USA).

\section{Ethical approval}

Ethical approval was given by the Human Research Ethics Committees of The University of Adelaide (H-202-2011) and the SA Department for Health and Ageing (463/07/2014).

\section{RESULTS}

\section{Descriptive}

A total of 7845 Salmonella spp. cases were reported from 1990 to 2012, of which $4412(56 \%)$ had an illness onset date in the warm season. Figure 1 shows the temporal distribution of daily Salmonella spp. 


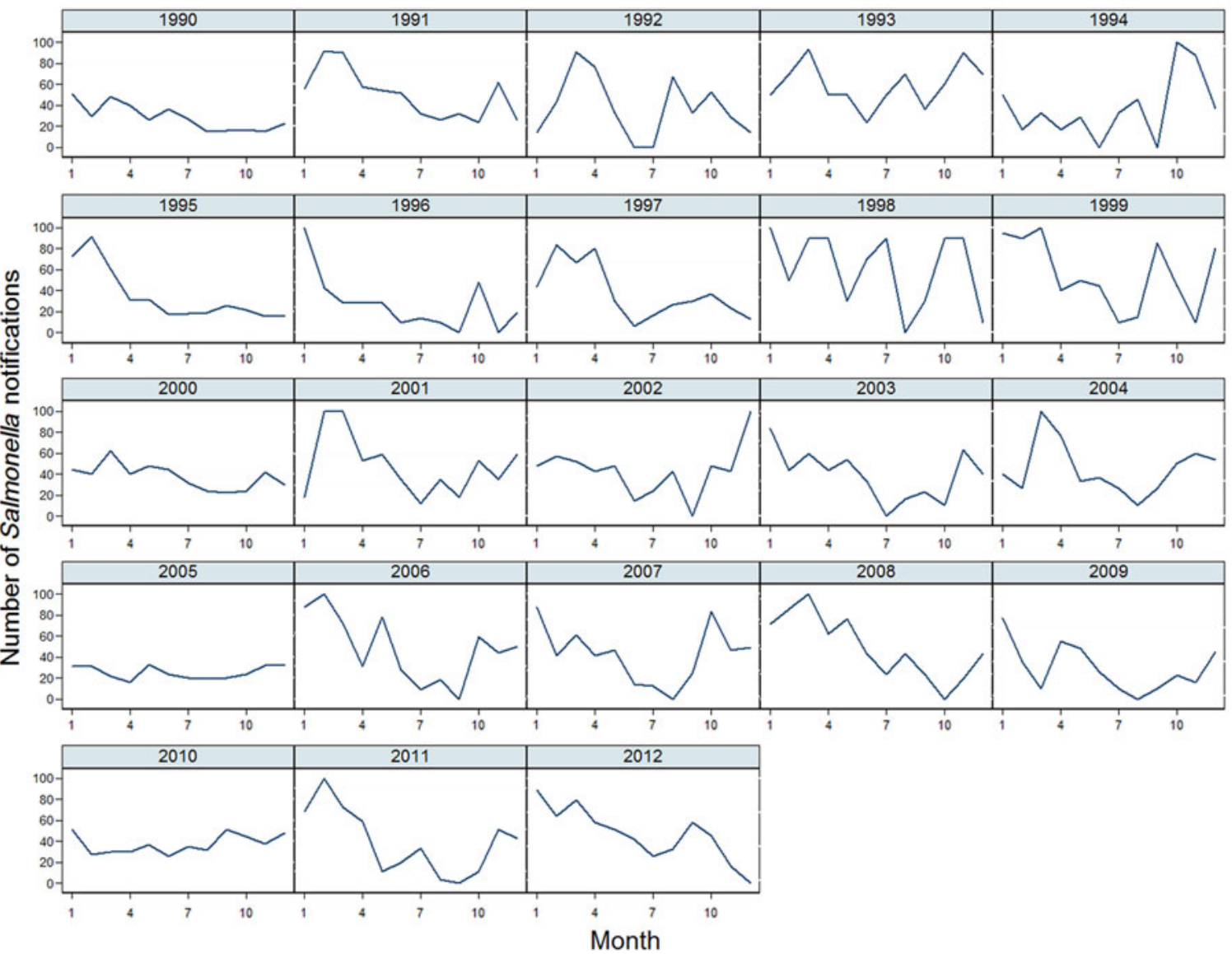

Fig. 1. Annual distribution of monthly notifications of Salmonella spp. infection, 1990-2012, Adelaide, South Australia.

notifications over the entire study period with peaks occurring in the warmer months. Table 1 presents summary statistics for the entire study period and for the warm season. The mean daily $T_{\max }$ during the warm seasons was $27^{\circ} \mathrm{C}$ (standard deviation $=$ $6 \cdot 1)$. The proportion of days during the warm season with a recorded $T_{\max }<20{ }^{\circ} \mathrm{C}$ was $6.5 \%$, and $>40{ }^{\circ} \mathrm{C}$ was $1 \%$. Little rainfall was recorded in the warm seasons and higher humidity was noted across the whole study period compared to the warm seasons.

There were 178 unique Salmonella spp. serotypes and phage types reported across the study period, with the five most frequently observed being: Salmonella enterica serovar Typhimurium phage type 9 ( $S$. Typhimurium PT9) $(n=422$ in warm season of 715 total in the study period; 59\%), Salmonella Infantis ( $S$. Infantis) $(n=229$ in warm season of 382 total; 60\%), S. Typhimurium PT108 $(n=209$ in warm season of 374 total; 56\%), S. Typhimurium PT44 ( $n=179$ in warm season of 297 total; 60\%) and $S$. Typhimurium PT135 $(n=178$ in warm season of 318 total; $56 \%$ ).
Daily $T_{\max }$ was positively correlated with the number of notifications for overall Salmonella spp. infection $(r=$ $0 \cdot 07)$. Weaker correlations were observed between $T_{\max }$ and $S$. Typhimurium PT9, PT135 and PT108 notifications. There was negligible correlation of $T_{\max }$ with $S$. Infantis and $S$. Typhimurium PT44 notifications.

We selected the optimal lag structure for $T_{\max }$ based on the maximum correlation coefficients identified from cross-correlation analyses for the final models as they improved the fit of the model. Lagged effects of $T_{\max }$ on the number of daily salmonellosis and serotype cases varied from 5 to 20 days with a lag of 14 days common across most of the five serotypes in the analyses. The AR order included in the Poisson regression models to control for AC of overall Salmonella spp. and serotype cases was based on PACF values. An AR structure of order 3 was included in the final model for overall salmonellosis; for serotypes, the optimal AR order ranged between 1 and 8 days. Daily $T_{\max }, T_{\max }^{2}$, the AR order of the number of salmonellosis and serotype cases and lag days for $T_{\max }$ based on the maximum correlation 
Table 1. Summary statistics for weather data, 1990-2012, Adelaide, South Australia

\begin{tabular}{|c|c|c|c|c|c|c|c|c|}
\hline \multirow[b]{2}{*}{ Weather variables } & \multicolumn{4}{|c|}{ Warm season* } & \multicolumn{4}{|l|}{ All seasons $\dagger$} \\
\hline & Mean (s.D.) & 25 th centile & 75th centile & 95th centile & Mean (S.D.) & 25 th centile & 75 th centile & 95th centile \\
\hline Daily $T_{\max }\left({ }^{\circ} \mathrm{C}\right)$ & $27 \cdot 0(6 \cdot 1)$ & $22 \cdot 0$ & $31 \cdot 0$ & $37 \cdot 7$ & $22 \cdot 4(6 \cdot 7)$ & $17 \cdot 0$ & $26 \cdot 6$ & $35 \cdot 3$ \\
\hline Daily $T_{\min }\left({ }^{\circ} \mathrm{C}\right)$ & $15 \cdot 2(4 \cdot 4)$ & $12 \cdot 2$ & $17 \cdot 7$ & $23 \cdot 5$ & $12 \cdot 3(4 \cdot 9)$ & $8 \cdot 9$ & $15 \cdot 1$ & $21 \cdot 5$ \\
\hline Daily rainfall (mm) & $0 \cdot 9(3 \cdot 5)$ & $0 \cdot 0$ & $0 \cdot 0$ & $5 \cdot 8$ & $1 \cdot 5(4 \cdot 2)$ & $0 \cdot 0$ & $0 \cdot 6$ & $8 \cdot 8$ \\
\hline $\begin{array}{l}\text { Daily RH, 09:00 } \\
\text { hours }(\%)\end{array}$ & $54 \cdot 0(18 \cdot 0)$ & $43 \cdot 0$ & $65 \cdot 0$ & $82 \cdot 0$ & $61 \cdot 4(19 \cdot 5)$ & $49 \cdot 0$ & $75 \cdot 0$ & $93 \cdot 0$ \\
\hline $\begin{array}{l}\text { Daily RH, 15:00 } \\
\text { hours (\%) }\end{array}$ & $39 \cdot 0(17 \cdot 0)$ & $27 \cdot 0$ & $49 \cdot 0$ & $69 \cdot 0$ & $46 \cdot 5(18 \cdot 6)$ & $34 \cdot 0$ & $58 \cdot 0$ & $80 \cdot 0$ \\
\hline
\end{tabular}

S.D., Standard deviation; $T_{\max }$, maximum temperature; $T_{\min }$, minimum temperature; RH, relative humidity.

* Salmonella spp. cases $(n=4412)$ notified in the warm season (October-March).

$\dagger$ Salmonella spp. cases $(n=7845)$ notified in the entire study period.

Table 2. Daily number of Salmonella spp. serotype and phage-type notifications reported in the warm season (October-March) per $1{ }^{\circ} \mathrm{C}$ increase in maximum temperature (T $\left.T_{\max }\right), 1990-2012$, Adelaide, South Australia

\begin{tabular}{|c|c|c|c|c|c|}
\hline Serotype & Variables & Lag day & IRR & $95 \% \mathrm{CI}$ & $P$ value \\
\hline \multirow[t]{2}{*}{ Salmonella spp. } & AR & 3 & $1 \cdot 061$ & $1.033-1.089$ & $0 \cdot 001$ \\
\hline & $T_{\max }$ & 14 & $1 \cdot 013$ & $1 \cdot 008-1 \cdot 019$ & $0 \cdot 001$ \\
\hline \multirow[t]{2}{*}{ S. Typhimurium PT9 } & $\mathrm{AR}$ & 1 & $1 \cdot 295$ & $1.094-1.533$ & $0 \cdot 003$ \\
\hline & $T_{\max }$ & 14 & $1 \cdot 037$ & $1 \cdot 020-1 \cdot 053$ & $0 \cdot 001$ \\
\hline \multirow[t]{2}{*}{ S. Typhimurium PT108 } & $\mathrm{AR}$ & 8 & $1 \cdot 640$ & $1 \cdot 224-2 \cdot 190$ & 0.001 \\
\hline & $T_{\max }$ & 5 & $1 \cdot 040$ & $1 \cdot 015-1 \cdot 065$ & $0 \cdot 001$ \\
\hline \multirow{2}{*}{ S. Typhimurium PT135 } & $\mathrm{AR}$ & 3 & $1 \cdot 647$ & $1 \cdot 126-2 \cdot 409$ & $0 \cdot 010$ \\
\hline & $T_{\max }$ & 16 & $1 \cdot 034$ & $1 \cdot 008-1 \cdot 061$ & $0 \cdot 009$ \\
\hline \multirow[t]{2}{*}{ S. Typhimurium PT44 } & $\mathrm{AR}$ & 3 & $1 \cdot 580$ & $1.280-1.951$ & $<0 \cdot 001$ \\
\hline & $T_{\max }$ & 20 & $1 \cdot 004$ & $0 \cdot 980-1 \cdot 028$ & $0 \cdot 718$ \\
\hline \multirow[t]{2}{*}{$S$. Infantis } & $\mathrm{AR}$ & 3 & 1.923 & $1.459-2.534$ & $<0.001$ \\
\hline & $T_{\max }$ & 14 & $1 \cdot 044$ & $1 \cdot 024-1 \cdot 064$ & $<0.001$ \\
\hline
\end{tabular}

AR, Autoregressive order; IRR, incidence rate ratio; CI, confidence interval.

$P$ value $(0.05$ significance level).

coefficients were included in the final time-series Poisson regression models.

\section{Effects of maximum temperature on salmonellosis}

As shown in Table 2 the number of salmonellosis notifications was related to the number occurring in the preceding 3 days. A $T_{\max }$ lag of 14 days was positively associated with the number of salmonellosis cases. A rise of $1{ }^{\circ} \mathrm{C}$ in $T_{\max }$ was associated with a $1.3 \%$ increase (IRR 1.013, 95\% CI 1.008-1.019) in the daily number of cases.

\section{Effects of maximum temperature on specific Salmonella spp. serotypes}

As demonstrated in Table 2, $S$. Infantis and all the $S$. Typhimurium phage types investigated with the exception of $S$. Typhimurium PT44 were associated with daily $T_{\max }$ increases. Each $1{ }^{\circ} \mathrm{C}$ increase in $T_{\max }$ was associated with a $3.7 \%$ increase in the daily number of $S$. Typhimurium PT9 cases (IRR $1 \cdot 037$, 95\% CI $1 \cdot 020-1 \cdot 053$ ) with a $T_{\max }$ lag in cases of 14 days. $T_{\max }$ at 5 days prior was positively associated with a $4 \%$ (IRR $1 \cdot 040$, 95\% CI $1 \cdot 015$ $1.065)$ increase in daily $S$. Typhimurium PT108 cases. For $S$. Typhimurium PT135 a $T_{\max }$ lag of 16 days was associated with a $3.4 \%$ (IRR $1 \cdot 034,95 \%$ CI $1.008-1.061$ ) increase in daily cases per $1{ }^{\circ} \mathrm{C}$ rise in temperature. For $S$. Infantis a $T_{\max }$ lag of 14 days in cases was observed with a $4.4 \%$ (IRR $1 \cdot 044$, 95\% CI 1.024-1.064) increase in daily cases per $1{ }^{\circ} \mathrm{C}$ rise in temperature. There was no significant association on daily $S$. Typhimurium PT44 notifications and $T_{\max }$. 

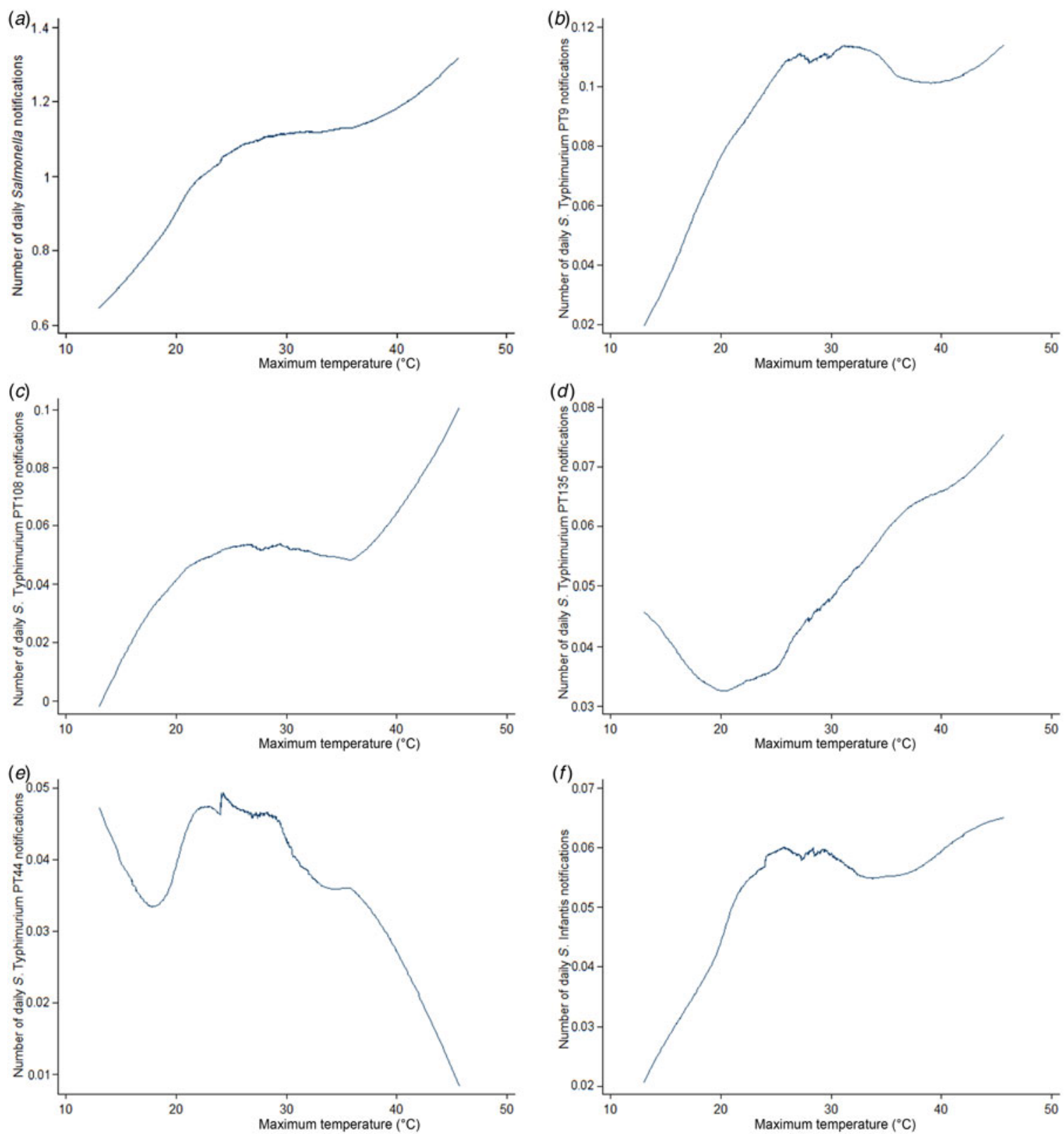

Fig. 2. Exposure-response relationships between maximum temperature and daily Salmonella spp. and serotype notifications, reported in the warm season (October-March), 1990-2012, Adelaide, South Australia. (a) Overall salmonellosis notifications, (b) $S$. Typhimurium PT9 notifications, (c) $S$. Typhimurium PT108 notifications, (d) $S$. Typhimurium PT135 notifications, (e) $S$. Typhimurium PT44 notifications, $(f) S$. Infantis notifications.

\section{Temperature thresholds}

Figure 2 demonstrates the exposure-response relationship between $T_{\max }$ and daily Salmonella spp. serotype and phage-type notifications during the warm season. The relationship between temperature and Salmonella spp. notifications changed above a certain threshold and we observed a broadly linear increase in cases with a rise in $T_{\max }$ above the threshold. We estimated a temperature threshold of $38.5{ }^{\circ} \mathrm{C}(95 \%$ CI $35 \cdot 9-41 \cdot 0$,
$P<0 \cdot 01)$ for overall salmonellosis cases as depicted in Figure $2 a$. For $S$. Typhimurium PT9 cases we detected a threshold of $39.2{ }^{\circ} \mathrm{C}(95 \%$ CI $34.5-43.8, P<0 \cdot 01)$ (Fig. $2 b$ ), for $S$. Typhimurium PT135 cases a threshold of $34.9{ }^{\circ} \mathrm{C}(95 \% \mathrm{CI} 29 \cdot 3-40 \cdot 4, P<0 \cdot 01)$ (Fig. $2 d$ ) and a threshold of $39.3{ }^{\circ} \mathrm{C}(95 \%$ CI $36.8-41 \cdot 7, P<$ $0 \cdot 01$ ) was detected for $S$. Typhimurium PT108 notifications (Fig. 2c). No thresholds for the effects of $T_{\max }$ 
on $S$. Typhimurium PT44 (Fig. 2e) and $S$. Infantis (Fig. 2f) cases were detected.

\section{DISCUSSION}

This study has identified that $S$. Infantis and certain phage types including $S$. Typhimurium PT9, PT135 and PT108 are more sensitive to the effects of temperature during the warm season. Our findings are in accordance with those from previous studies in that salmonellosis cases increase with warmer temperature. However, as addressed in the limitations of some studies [14, 19], serotypes could have different sensitivity to climate variables and should be examined to account for such effects. Two studies considered the effect of temperature on $S$. Typhimurium $[20,21]$, a common serotype isolated from humans and farm animals in Australia as well as in other countries [29], and $S$. Enteritidis, which is not commonly reported in Australia. However, the association was made at the serotype level with no further examination of significance at the phage-type level. Our study addressed these gaps by examining whether different serotypes and more distinctively, if phage types are likely to be affected by ambient temperature. Thus, our work contributes not only to the knowledge gap in this area, but also to public health practices in disease control and prevention.

We found an increase in daily counts of $1.3 \%$ on the overall number of salmonellosis cases per $1{ }^{\circ} \mathrm{C}$ rise in maximum temperature, with greater observed increases ranging from $3 \cdot 4 \%$ to $4 \cdot 4 \%$ in cases depending on the serotype and phage type. Other studies examining the effect of temperature on salmonellosis found increases of $1-15 \%$ of cases per $1{ }^{\circ} \mathrm{C}$ rise in temperature [11-22]. However, these estimates are based on Salmonella spp. counts aggregated by week or month. We used daily disease notification with date of onset from surveillance data which may be more sensitive in detecting variability in temperature than weekly or monthly aggregated data as daily Salmonella spp. notifications should align better with climate data [30].

$S$. Infantis and all of the $S$. Typhimurium phage types in our analyses, with the exception of $S$. Typhimurium PT44, were significantly associated with an increase in cases linked to temperature in the warmer months. Although $60 \%$ of cases were reported in the warm season, $S$. Typhimurium PT44 did not appear to be associated with $T_{\max }$. Some Salmonella spp. strains may be better acclimatized to higher temperature which could be the case for this phage type [31]. However, the number of notifications reported during the warm seasons was sparse and the results should be interpreted with caution.

We also found that the effects of $T_{\max }$ on daily Salmonella spp. notifications, both overall and for $S$. Infantis and $S$. Typhimurium phage types were delayed by up to 14 days which supports the findings from a previous study conducted in Adelaide [18]. Further to this a lag of 1 month was found in five Australian states, including SA [12]. Varying lags have been detected in other studies with temperature peaking at 1 week [20], 2 weeks [17], 1 month [11, $14,15,22]$ and up to 2 months [13, 16, 18]. A lag of temperature on Salmonella spp. notifications varies between geographical regions because of location specific factors including other relevant climatic conditions [19]. We found an overall $T_{\max }$ lag of 14 days in Salmonella spp. serotype and phage-type notifications in our study with the exception of $S$. Typhimurium PT108 where we observed a shorter lag of 5 days. Our findings associated with lag effects of temperature on serotype notifications is similar to other studies in that $T_{\max }$ with 1- and 2-week lags, persisting up to 5 weeks were positively associated with $S$. Typhimurium and $S$. Enteritidis cases [20, 21]. The incubation period for Salmonella spp. infection is estimated between $8 \mathrm{~h}$ and $72 \mathrm{~h}$ and lag effects of temperature can also be associated with different incubation periods [24]. This difference depends on the serotype and phage type and is influenced by infectious dose, virulence, transmission route and individual susceptibility [32]. Identification of which of the commonly notified serotypes and phage types are affected by temperature in the warm season, including lag effects is important because of the implications for foodborne disease prevention.

The prevalence of Salmonella spp. serotypes is influenced by geographical and epidemiological differences thus affecting transmission routes [33]. As stated earlier the transmission pathways for Salmonella spp. serotypes and phage types may be a reason for the differences in lag effects found across our study and those reported previously [21]. Our finding of a shorter effect of $T_{\max }$ on $S$. Typhimurium PT108 cases could also point to differences in transmission pathways associated with this particular phage type. Lag effects are important as they provide an indication where food contamination could have occurred. Short lag times with temperature points to food contamination closer to the time of consumption, whereas longer 
time lags could indicate effects at the production processing stages [21].

Findings from our study of $T_{\max }$ lags of 5 and 14 days is suggestive of influences occurring closer to the primary food production stages which has implications for public health interventions and food safety regulation. Pathogens can enter and contaminate the food chain from beginning (farm) to end (fork). At the primary production stage contamination may occur through the use of manure and water to fertilize and irrigate fresh fruit and vegetables [34]. Because Salmonella spp. can survive outside of animal or human hosts, higher ambient temperatures may enhance replication in manure. This may facilitate transmission between animals, particularly in conditions associated with intensive farming and overcrowding [1]. Likewise, higher temperatures can lead to livestock animals becoming stressed with increases in the rates of multiplication and subsequent shedding of the bacteria into the environment [10].

Our findings suggest that Salmonella spp. serotypes and particular phage types are affected by temperature [25]. This questions the role of environmental pathways in salmonellosis infection. Some Salmonella spp. serotypes and phage types may be more endemic in the environment and not necessarily associated with food, but with other environmental exposures, such as warmer ambient temperature. For example, in Tasmania, Australia, $S$. Mississippi is endemic suggesting that transmission is associated with environmental exposure and not food [26]. Understanding the mechanisms of the relationship of temperature on transmission of Salmonella spp. infection may point to possible sources of those strains, such as host and reservoirs and environmental conditions [25], hence the need for targeted public health messages and producer-related policies to reduce incidence of and consequent burden associated with disease.

The serotypes and phage types in this study are common in Australia and are isolated from human and non-human sources [29]. Non-human sources from farmed animals include cows, sheep, pigs and poultry. The serotypes and phage types in our analyses were commonly isolated from broiler chickens ( $S$. Infantis, $S$. Typhimurium PT135), eggs ( $S$. Typhimurium PT135 and PT108) and egg shed drag swabs (in order of decreasing frequency, $S$. Typhimurium PT135, PT108, PT9 and $S$. Infantis). $S$. Typhimurium PT9, PT108 and PT135 were isolated from cattle, followed by sheep and poultry [35]. Although we excluded outbreaks from the analyses, the serotypes in our study isolated from farmed animals are also linked to foodborne related outbreaks associated with eggs and chicken and this suggests transmission via contaminated food [31, 36-40]. Food contamination and pathogen transmission is highly complex as there are many causal pathways which involve several mechanisms [25].

Overall salmonellosis cases increased when a higher temperature threshold of $>38^{\circ} \mathrm{C}$ (which is above the 95th percentile for daily $T_{\max }$ in the warm season as shown in Table 1) was reached. $S$. Typhimurium PT9, PT108 and PT135 notifications increased when the threshold was between $34 \cdot 9^{\circ} \mathrm{C}$ and $39 \cdot 3{ }^{\circ} \mathrm{C}$. Temperature thresholds examined in other studies found none $[14,15,19]$, while in another study the relationships between Salmonella spp. cases and temperature was linear above a certain threshold [20]. Detection of thresholds is important in disease control and prevention because there may be excess notifications if temperature rises above a certain level. We identified thresholds above the 95th percentile and with increasing frequency in hot weather, especially heatwaves we may expect to see an increase in Salmonella spp. cases.

Given that the incidence of Salmonella spp. infection is likely to increase with rises in temperature predicted with climate change, extreme high temperatures such as those detected with the thresholds highlights the importance of this study and the need for foodborne disease prevention during the warmer months.

Our study has several limitations. Salmonella spp. infection is underreported given its reliance on a passive disease surveillance notification system [6]. However, it is unlikely that underreporting will affect estimates of the association between temperature and increased risk of salmonellosis. In addition, underreporting is believed to have remained relatively stable over the study period [18]. We were unable to exclude cases that travelled outside of the study area prior to onset of illness because the data were not fully captured in the disease notification surveillance system. Only a small proportion of cases are expected to travel and inclusion of cases that travelled is unlikely to have any tangible effect on the estimates. The proportion of Salmonella spp. cases notified in the warm season was $56 \%$ overall and about $60 \%$ for the five serotype and phage types. The proportion of cases could be low in the context of our hypothesis of an association between temperature in the warm season and increased Salmonella spp. cases and warrants further examination of the potential for excess cases attributed to high temperatures.

The fact that Salmonella spp. can multiply in food, and the examination of any $T_{\max }$ lags in cases provides a useful framework through which to investigate the 
points in the food chain at which risks alter with temperature change [24], as this has implications for disease prevention and control. Where the risk occurs in relation to effects on primary production, food processing and/or food handling by consumers [1] has implications for food safety, particularly food preparation, handling and storage at the time of consumption and could be incorporated as part of an early warning system for public health awareness. Similarly, effects introduced at the stage of primary production will require industry and government intervention with monitoring and enforcement of industry standards an important mechanism for prevention [41]. In addition, increased cases of Salmonella spp. infection in the warm season could be associated with human behaviour. Changes in eating patterns and behaviour such as eating out, consumption of ready-to-eat foods, outdoor eating and barbequing may lead to people's exposure to Salmonella spp. infection $[1,13]$. The results from our study indicate a need for tailored preventive control measures that account for different Salmonella spp. serotypes and phage types and point to transmission routes that may be associated with human behaviour, environment and climatic factors such as warmer ambient temperature.

\section{CONCLUSION}

With populations worldwide exposed to variability in weather patterns and increasingly warm temperatures, the burden of diarrhoeal diseases, including Salmonella spp. infection is likely to increase if climate change continues as projected [7]. Knowledge about the delayed effect of temperature on foodborne infection is important, because contamination can occur at any point along the food chain, thus influencing actions in foodborne disease prevention. Additional studies are needed to contribute knowledge about different Salmonella spp. serotypes and phage types and the relationship with temperature and links to nonhuman sources, environmental exposure and transmission as well as foodborne transmission pathways. Together with the findings reported here, this information will contribute to an understanding of possible causal mechanisms associated with foodborne infection and temperature that plays important roles for informing public health policy and intervention.

\section{ACKNOWLEDGEMENTS}

The authors thank staff from the Disease Surveillance and Investigation Section, Communicable Disease
Control Branch, Department for Health and Ageing (SA Health) for their support in conducting this study.

\section{DECLARATION OF INTEREST}

None.

\section{REFERENCES}

1. Hall GV, D'Souza RM, Kirk MD. Foodborne disease in the new millennium: out of the frying pan and into the fire? Medical Journal of Australia 2002; 177: 614-618.

2. Majowicz SE, et al. The global burden of nontyphoidal Salmonella gastroenteritis. Clinical Infectious Diseases 2010; 50: 882-889.

3. Kirk M, et al. Foodborne illness, Australia, circa 2000 and circa 2010. Emerging Infectious Diseases 2014; 20: 1857-1864.

4. Lalor K. Salmonella surveillance in Australia and Victoria. In: Proceedings of the Environmental Health Professionals Australia. Inaugural National Symposium. Melbourne, Victoria: Environmental Health Professionals Australia, 2012, pp. 2.

5. The OzFoodNet Working Group. Monitoring the incidence and causes of diseases potentially transmitted by food in Australia: Annual Report of the OzFoodNet Network, 2009. Communicable Diseases Intelligence 2010; 31: 345-365.

6. Hall G, et al. Estimating foodborne gastroenteritis, Australia. Emerging Infectious Diseases 2005; 11: 1257-1264.

7. Smith K, et al. Human health: impacts, adaptation, and co-benefits. United Kingdom and New York, NY, USA: Intergovernmental Panel on Climate Change; 2014. Climate Change 2014: Impacts, Adaptation, and Vulnerability Part A: Global and Sectoral Aspects Contribution of Working Group II to the Fifth Assessment Report of the Intergovernmental Panel on Climate Change.

8. Department of Environment and Natural Resources. Regional climate change projections: Adelaide and Mount Lofty Ranges, South Australia; 2010.

9. Deo RC, et al. On Australian heat waves: time series analysis of extreme temperature events in Australia, 1950-2005. In: MODSIM 2007 International Congress on Modelling and Simulation. New Zealand: The Modelling and Simulation Society of Australia and NZ Inc., 2007, p. 10.

10. Miraglia M, et al. Climate change and food safety: an emerging issue with special focus on Europe. Food and Chemical Toxicology 2009; 47: 1009-1021.

11. Britton E, et al. Positive association between ambient temperature and salmonellosis notifications in New Zealand, 1965-2006. Australian and New Zealand Journal of Public Health 2010; 34: 126-129.

12. D'Souza RM, et al. Does ambient temperature affect foodborne disease? Epidemiology 2004; 15: 86-92.

13. Fleury M, et al. A time series analysis of the relationship of ambient temperature and common bacterial enteric 
infections in two Canadian provinces. International Journal of Biometeorology 2006; 50: 385-391.

14. Grjibovski AM, et al. Climate variations and salmonellosis in northwest Russia: a time-series analysis. Epidemiology and Infection 2013; 141: 269-276.

15. Grjibovski AM, Kosbayeva A, Menne B. The effect of ambient air temperature and precipitation on monthly counts of salmonellosis in four regions of Kazakhstan, Central Asia, in 2000-2010. Epidemiology and Infection 2014; 142: 608-615.

16. Lal A, et al. Climate variability, weather and enteric disease incidence in New Zealand: time series analysis. PLOS ONE 2013; 8: e83484.

17. Naumova EN, et al. Seasonality in six enterically transmitted diseases and ambient temperature. Epidemiology and Infection 2007; 135: 281-292.

18. Zhang Y, Bi P, Hiller J. Climate variations and salmonellosis transmission in Adelaide, South Australia: A comparison between regression models. International Journal of Biometeorology 2008; 52: 179-187.

19. Zhang Y, Bi P, Hiller JE. Climate variations and Salmonella infection in Australian subtropical and tropical regions. Science of the Total Environment 2010; 408: 524-530.

20. Kovats RS, et al. The effect of temperature on food poisoning: a time-series analysis of salmonellosis in ten European countries. Epidemiology and Infection 2004; 132: 443-453.

21. Lake IR, et al. A re-evaluation of the impact of temperature and climate change on foodborne illness. Epidemiology and Infection 2009; 137: 1538-1547.

22. Ravel A, et al. Seasonality in human salmonellosis: assessment of human activities and chicken contamination as driving factors. Foodborne Pathogens and Disease 2010; 7: 785-794.

23. Rabsch W. Salmonella Typhimurium phage typing for pathogens. In: Schatten H, Eisenstark A, eds. Salmonella: Totowa, NJ: Humana Press, 2007, pp. 177-211.

24. Craig D, Batholomaeus A. Agents of foodborne illness. A technical series summarising key information on microorganisms associated with foodborne illness. Canberra: Food Standards Australia New Zealand; 2011.

25. Strawn LK, et al. Distributions of salmonella subtypes differ between two U.S. produce-growing regions. Applied and Environmental Microbiology 2014; 80: 3982-3991.

26. Ashbolt R, Kirk MD. Salmonella Mississippi infections in Tasmania: the role of native Australian animals and untreated drinking water. Epidemiology and Infection 2006; 134: 1257-1265.

27. Xiang $\mathbf{J}$, et al. Association between high temperature and work-related injuries in Adelaide, South Australia,
2001-2010. Journal of Occupational and Environmental Medicine 2014; 71: 246-252.

28. Hansen A, et al. The effect of heat waves on mental health in a temperate Australian city. Environmental Health Perspectives 2008; 116: 1369-1375.

29. Pang S, et al. Genomic diversity and adaptation of Salmonella enterica serovar Typhimurium from analysis of six genomes of different phage types. BMC Genomics 2013; 14: 718.

30. Bi P, et al. Weather and notified Campylobacter infections in temperate and sub-tropical regions of Australia: an ecological study. Journal of Infection 2008; 57: 317-323.

31. Lim JA, Lee DH, Heu S. The interaction of human enteric pathogens with plants. Plant Pathology 2014; 30: 109-116.

32. Naumova EN, MacNeill IB. Time-distributed effect of exposure and infectious outbreaks. Environmetrics 2009; 20: 235-248.

33. Vally H, et al. Proportion of illness acquired by foodborne transmission for nine enteric pathogens in Australia: an expert elicitation. Foodborne Pathogens and Disease 2014; 11: 727-733.

34. Edwards F, et al. Climate change adaptation at the intersection of food and health. Asia-Pacific Journal of Public Health 2011; 23: 91S-104.

35. Microbiological Diagnostic Unit. National Enteric Pathogens Surveillance Scheme Non-Human Annual Report 2012: Microbiological Diagnostic Unit, The University of Melbourne, 2012.

36. Denehy EJ, et al. Outbreak of Salmonella typhimurium phage type 44 infection among attendees of a wedding reception, April 2009. Communicable Diseases Intelligence 2011; 35: 192-196.

37. Fearnley E, et al. Salmonella in chicken meat, eggs and humans; Adelaide, South Australia, 2008. International Journal of Food Microbiology 2011; 146: 219-227.

38. Gole VC, et al. Effect of egg washing and correlation between eggshell characteristics and egg penetration by various Salmonella Typhimurium strains. PLoS ONE 2014; 9: e90987.

39. Najjar Z, et al. An outbreak of Salmonella Infantis gastroenteritis in a residential aged care facility associated with thickened fluids. Epidemiology and Infection 2012; 140: 2264-2272.

40. Stephens N, Coleman D, Shaw K. Recurring outbreaks of Salmonella typhimurium phage type 135 associated with the consumption of products containing raw egg in Tasmania. Communicable Diseases Intelligence 2008; 32: 466-468.

41. Bambrick HJ, et al. Climate change and health in the urban environment: adaptation opportunities in Australian cities. Asia-Pacific Journal of Public Health 2011; 23: 67S-79. 